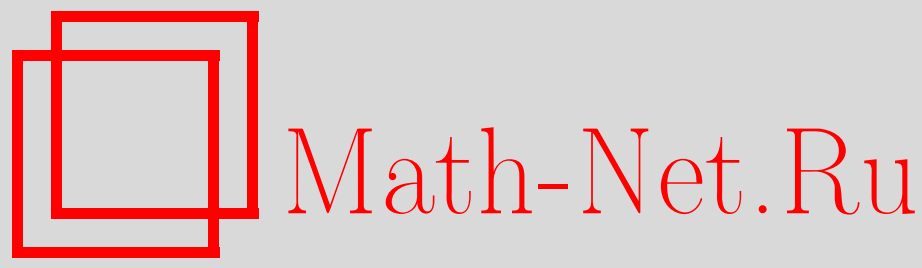

А. М. Зубков, Изоморфизмы между множествами вероятностных распределений, Теория вероятн. и ее примен., 1999, том 44, выпуск 4, 861-865

DOI: https://doi.org/10.4213/tvp1071

Использование Общероссийского математического портала Math-Net.Ru подразумевает, что вы прочитали и согласны с пользовательским соглашением

http: //www . mathnet.ru/rus/agreement

Параметры загрузки:

IP : 3.89 .185 .249

26 апреля 2023 г., 16:31:59

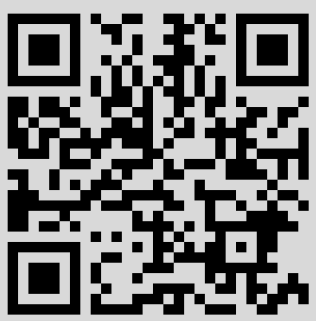


4. Прохоров ЮО.В. Об одной лохальной теореме. - В сб.: Предельные теоремы теории вероятностей. Ташкент: Изд-во АН УзССР, 1963, с. 75-80.

5. Бхаттачария $P$. H., Ранга $Р$ ао $P$. Аппроксимация нормальным распределением и асимптотические разложения. М.: Наука, 1982, 286 с.

6. Ушаков Н.Г. Неравенства для характеристических функпий. - В сб.: Методы решения задач математической физики и их программное обеспечение. М.: Изд-во Моск. ун-та, 1984, с. 50-53.

7. Феллер В. Введение в теорию вероятностей и ее приложения. Т. 2. М.: Мир, 1984, $752 \mathrm{c}$.

8. Ushakov N. G. Lower and upper bounds for characteristic functions. - J. Math. Sci., 1997 , v. 84, № 3, p. 1179-1189.

9. Korolev V. Yu., Zhukov Yu. V. On the rate of convergence in the local limit theorem for densities. - J. Math. Sci., 1998, v. 91, № 3, p. 2931-2941.

Поступила в редакцию 19.I.1998

(C) $1999 \mathrm{r}$.

ЗУБКОВ А. M.*

\title{
ИЗОМОРФИЗМЫ МЕЖДУ МНОЖЕСТВАМИ ВЕРОЯТНОСТНЫХ РАСПРЕДЕЛЕНИЙ ${ }^{1)}$
}

\begin{abstract}
Указаны способы построения изоморфизмов между полугруппами вероятностных распределений (со сверткой как полугрупповой операцией), в частности, между множеством всех вероятностных распределений на неотрицательной полуоси и некоторой совокупностью распределений на множестве неотрицательных целых чисел. Аналогичные изоморфизмы указаны для векторного случая и для множеств распределений, соответствуюших неубывающим процессам с независимыми приращениями.
\end{abstract}

Ключевые слова и фразы: полугруппы вероятностных распределений, неотрицательные случайные величины, изоморфизмы между множествами распределений.

В настоящей работе используется следуюшее соображение. Пусть $P$ - семейство всех вероятностных распределений на $\mathbf{R}_{+}=[0, \infty)$. Обозначим через $\delta_{x}$ распределение, сосредоточенное в точке $x \in \mathbf{R}_{+}$, и через $D=\left\{\delta_{x}, x \in \mathbf{R}_{+}\right\}$семейство вырожденных распределений на $\mathbf{R}_{+}$. Относительно операции свертки семейства $P$ и $D$ являются коммутативными полугруппами; более того, для однопараметрического семейства $D$ полугрупповая структура, порожденная сверткой, согласована со структурой аддитивной полугруппы $\mathbf{R}_{+}: \delta_{x} * \delta_{y}=\delta_{x+y}$ при любых $x, y \in \mathbf{R}_{+}$.

Любое вероятностное распределение $F \in P$ можно представить в виде смеси распределений из семейства $D$ :

$$
F=\int_{0}^{\infty} \delta_{x} F(d x)
$$

Пусть, далее, $B=\left\{B_{x}, x \in \mathbf{R}_{+}\right\}-$любое семейство распределений (например, на $\mathbf{R}^{d}$ ), образующее полугруппу относительно операции свертки, причем

* Математический институт им. В. А. Стеклова РАН, ул. Губкина, 8, 117966 Москва, ГСП-1, Россия; e-mail: zubkov@genesis.mi.ras.ru

1) Выполнение работы частично финансировалось Российским фондом фундаментальных исследований (проект 96-01-00531) и Советом по грантам Президента РФ и государственной поддержке ведуших научных школ (проект 96-15-96092). 
$B_{x} * B_{y}=B_{x+y}$ при любых $x, y \in \mathbf{R}_{+}$. Такая полугруппа соответствует совокупности распределений случайного однородного по времени процесса $\beta(x), x \in \mathbf{R}_{+}$, c независимыми приращениями; семейство $D$ соответствует детерминированному процессу $\beta(x) \equiv x$. Отображение $\Pi_{B}$, переводящее распределение $F \in P$ в распределение

$$
\Pi_{B} F=\int_{0}^{\infty} B_{x} F d(x)
$$

значения $\beta(\tau)$ (где $\tau$ - случайная величина с распределением $F$, не зависящая от процесса $\beta$ ), обладает следуюшим свойством: для любых распределений $F, G \in P$ (отождествляемых с соответствуюшими функциями распределения)

$$
\begin{aligned}
\Pi_{B}(F * G) & =\int_{0}^{\infty} B_{x} d(F * G)(x)=\int_{0}^{\infty} B_{x} d\left(\int_{0}^{x} F(x-y) d G(y)\right) \\
& =\int_{0}^{\infty} \int_{0}^{x} B_{x-y} * B_{x} d F(x-y) d G(y)=\int_{0}^{\infty} \int_{0}^{\infty} B_{x} * B_{y} d F(x) d G(y) \\
& =\int_{0}^{\infty} B_{x} d F(x) * \int_{0}^{\infty} B_{y} d G(y)=\Pi_{B} F * \Pi_{B} G .
\end{aligned}
$$

Таким образом, $\Pi_{B} P$ - полугруппа и отображение $\Pi_{B}$ является гомоморфизмом полугрупп П и $\Pi_{B} P$.

Формула (2) эквивалентна очевидному утверждению: если $\tau$ и $\eta$ - независимые случайные величины, не зависяшие от однородного процесса с независимыми приращениями $\beta$, то распределения $\beta(\tau+\eta)$ и $\beta(\tau)+\beta(\eta)$ совпадают. В случае, когда $B-$ полугруппа распределений на множестве целых чисел (она соответствует распределениям сложного пуассоновского процесса, см. [1]-[3]), отображение $\Pi_{B}$ яляется гомоморфизмом между множеством $P$ всех вероятностных распределений на $\mathbf{R}_{+}$и множеством $\Pi_{B} P$ распределений на множестве целых чисел. Мы покажем, что во многих случаях такое отображение $\Pi_{B}$ оказывается не только гомоморфизмом, но и изоморфизмом, откуда следует, в частности, что множество всех распределений на $\mathbf{R}_{+}$эквивалентно множеству, состоящему из распределений на множестве целых неотрицательных чисел.

Теорема 1. Пусть $B=\left\{B_{x}, x \in \mathbf{R}_{+}\right\}$- семейство распределений однородного случайного прочесса $\beta(x)$ с независимыми приращениями, $\mathbf{P}\{\beta(0)=0\}=1$, причем существует такое $a>0$, что для всех $x \in \mathbf{R}_{+}$преобразование Лапласа $\mathbf{E} e^{-\boldsymbol{x}(x)}$ аналитично в полосе $0<\operatorname{Re} z<a$ и при всех $z \in(0, a), x \in \mathbf{R}_{+}$справедливо неравенство $0<\mathbf{E} e^{-\boldsymbol{x} \beta(x)}<1$. Тогда определенное формулой (1) отображение $\Pi_{B}$ ввляется изоморфизмом между полугруппой $P$ всех распределений на $\mathbf{R}_{+}$ и ее образом - полугруппой $\Pi_{B} P$, и непрерывно относительно слабой сходимости распределений.

Д о к а з а т е л с т в о. В силу (2) $\Pi_{B} P$ яляется полугруппой, а отображение $\Pi_{B}$ - гомоморфизмом полугрупп. Докажем взаимную однозначность отображения $\Pi_{B}$.

Пусть $\gamma$ - неотрицательная случайная величина с распределением $F \in P$; ее преобразование Лапласа $f_{\gamma}(z)=\mathbf{E} e^{-z \gamma}$ определено при $\operatorname{Re} z \geqslant 0$ и является в этой области аналитической функцией. В силу (1) распределение $\Pi_{B} F-$ это распределение случайной величины $\nu=\beta(\gamma)$, где случайная величина $\gamma$ и случайный процесс $\beta(x)$ независимы. По свойству однородных процессов с независимыми прирашениями $\mathbf{E} e^{-z \beta(x)}=\left(\mathbf{E} e^{-z \beta(1)}\right)^{x}, x \in \mathbf{R}_{+}$, и, значит, при тех действительных $z$, при которых $0<\mathbf{E} e^{-x \beta(x)}<1$ для всех $x \geqslant 0$, имеет место равенство

$$
\mathbf{E} e^{-z \nu}=\mathbf{E} e^{-z \beta(\gamma)}=\mathbf{E} \mathbf{E}\left\{e^{-x \beta(\gamma)} \mid \gamma\right\}=\mathbf{E}\left(\mathbf{E} e^{-z \beta(1)}\right)^{\gamma}=f_{\gamma}\left(-\ln \mathbf{E} e^{-x \beta(1)}\right) .
$$

Из условий теоремы следует, что преобразование Лапласа $\mathrm{Ee}^{-z \nu}$ случайной величины $\nu=\beta(\gamma)$ определено в полосе вида $\operatorname{Re} z \in(0, a), a>0$, и является в ней аналитической функцией. В силу (3) значения аналитической функции $\mathrm{Ee}^{-\boldsymbol{z \gamma}}$ на бесконечном множестве точек (отрезке $[0, a]$ действительной оси) функционально связаны со значениями аналитической функции $f_{\gamma}(z)$ на отрезке, образованном значениями 
$-\ln \mathbf{E} e^{-x \beta(1)}, z \in[0, a]$, и, следовательно (см. [4, с. 104]), каждая из этих функций однозначно определяет другую во всей области ее аналитичности. Отсюда и из взаимной однозначности соответствия между действительными случайными величинами и их преобразованиями Лапласа следует, что отображение $\Pi_{B}$ взаимно однозначно.

Непрерывность отображения $\Pi_{B}$ относительно слабой сходимости распределений следует из тождества (3) и теоремы о непрерывности соответствия между распределениями и их преобразованиями Лапласа [5, с. 486].

3 а м е ч а н и е 1. Если однородный случайный процесс с независимыми приращениями $\beta(x)$ принимает только целочисленные значения, то равенство (3) можно переписать в терминах производящих функций:

$$
\mathbf{E} s^{\nu}=\mathbf{E} s^{\beta(\gamma)}=\mathbf{E} \mathbf{E}\left\{s^{\beta(\gamma)} \mid \gamma\right\}=\mathbf{E}\left(\mathbf{E} s^{\beta(1)}\right)^{\gamma},
$$

и при выполнении условий теоремы 1 отображение $\Pi_{B}$ устанавливает изоморфизм между множеством распределений на $\mathbf{R}_{+}$и его подмножеством, содержашимся в множестве распределений на $\mathbf{Z}_{+}=\{0,1, \ldots\}$.

3 а м е ч а н и е 2. Рассматривая вместо $\beta(x)$ процесс $-\beta(x)$, можно переформулировать теорему 1 для случая, когда преобразование Лапласа $\mathrm{Ee}^{-\boldsymbol{x} \beta(x)}$ аналитично в полосе $-a<\operatorname{Re} z<0$.

Разумеется, вместо семейства распределений $B$, зависяших от параметра $x \in \mathbf{R}_{+}$, можно рассматривать семейства распределений, параметризованные элементами какой-нибудь другой коммутативной полугруппы $G$. Конструкцию, аналогичную (1), можно реализовать с помощью любого нетривиального семейства распределений $B=\left\{B_{x}, x \in G\right\}$ на коммутативной полугруппе $H$, удовлетворяющего условиям

$$
B_{x+y}=B_{x} * B_{y} \quad \text { для любых } x, y \in G .
$$

(Условие (5) может оказаться весьма ограничительным, если в полугруппе $G$ допустимы равенства типа $x+y_{1}+\cdots+y_{k}=x$ при ненулевых $y_{1}, \ldots, y_{k}$. В настоящей статье мы рассмотрим только случай, когда $G=\mathbf{R}_{+}^{g}$.)

Пусть $P_{G}$ - семейство распределений случайных величин, принимающих значения в полугруппе $G$, и $F \in P_{G}$; формула

$$
\Pi_{B} F=\int_{G} B_{x} F(d x)
$$

определяет отображение $\Pi_{B}$, переводящее распределения $F \in P_{G}$ в распределения на $H$. Почти дословное повторение рассуждений, использованных в (2) (интегралы по $\mathbf{R}_{+}$в (2) следует заменить интегралами по $G$, а интегралы от 0 до $x$ - интегралами по множеству $\{y \in G: y-x \in G\}$ ), показывает, что отображение $\Pi_{B}$ есть гомоморфизм множеств распределений на $G$ и на $H$, образуюших полугруппы относительно операции свертки.

При $G=\mathbf{R}_{+}^{g}$ и $H=\mathbf{R}^{h}$ описание общего вида семейства $B$, удовлетворяющего условиям (5), сводится к одномерному случаю, так как тогда $B_{\mathbf{X}}=B_{x_{1}} \mathrm{e}_{1} * \cdots * B_{x_{g}} \mathbf{e}_{g}$ при любом $\mathbf{x}=\left(x_{1}, \ldots, x_{g}\right) \in \mathbf{R}_{+}^{g}$, где $\mathbf{e}_{1}, \ldots, \mathbf{e}_{g}$ - единичные векторы координатных осей, а каждое семейство $\left\{B_{x} \mathbf{e}_{i}, x \in \mathbf{R}_{+}\right\}, i=1, \ldots, g$, яляется семейством распределений однородного процесса с независимыми приращениями и со значениями в $\mathbf{R}^{h}$. Сформулируем полученное утверждение в виде леммы.

Лемма. Любое семейство распределений $B=\left\{B_{\mathbf{X}}, \mathbf{x} \in \mathbf{R}_{+}^{g}\right\}$ на $\mathbf{R}^{h}$, удовлетворяющее условиям $B_{\mathbf{X}} * B_{\mathbf{y}}=B_{\mathbf{X}}+\mathbf{y}$ при любых $\mathbf{x}, \mathbf{y} \in \mathbf{R}_{+}^{g}$, определяется $g$ независимыми однородными прочессами $\left\{\beta_{i}(t), t \geqslant 0\right\}, i=1, \ldots, g$, со зяачениями в $\mathbf{R}^{h}$ и с независимыми прирачениями: распределение $B_{\mathbf{X}}, \mathbf{x}=\left(x_{1}, \ldots, x_{g}\right)$, совпадает $c$ распределением суммы $\beta_{1}\left(x_{1}\right)+\cdots+\beta_{g}\left(x_{g}\right)$ при любых $x_{1}, \ldots, x_{g} \geqslant 0$.

Для семейства $B$, удовлетворяюшего условиям (5), отображение $\Pi_{B}$ переводит распределение $F$ вектора $\gamma=\left(\gamma_{1}, \ldots, \gamma_{g}\right) \in \mathbf{R}_{+}^{g}$ в распределение случайного вектора $\beta_{1}\left(\gamma_{1}\right)+\cdots+\beta_{g}\left(\gamma_{g}\right) \in \mathbf{R}^{h}$, и справедлив аналог теоремы 1 .

Чтобы упростить доказательство, будем считать, что $g=h$.

Теорема 2. Пусть $B=\left\{B_{\mathbf{X}}, \mathbf{x} \in \mathbf{R}_{+}^{g}\right\}-$ семейство распределений на $\mathbf{R}^{g}: B_{\mathbf{X}}$ при каждом $\mathrm{x}=\left(x_{1}, \ldots, x_{g}\right) \in \mathbf{R}_{+}^{g}-$ распределение вектора $\beta_{1}\left(x_{1}\right)+\cdots+\beta_{g}\left(x_{g}\right)$, 
где $\beta_{1}(x), \ldots, \beta_{g}(x)$ ( $\left.x \in \mathbf{R}_{+}\right)$- жезависимые однородные случайные прочессы с независимьми прирачениями и зяачениями в $\mathbf{R}^{g}, \mathbf{P}\left\{\beta_{k}(0)=0\right\}=1(k=1, \ldots, g)$. Пусть существует такой вектор $\mathbf{a}=\left(a_{1}, \ldots, a_{g}\right) \in \mathbf{R}^{g}$ с положительными координатами, ито преобразования Лапласа $\mathbf{E} \exp \left\{-\left(\mathbf{z}, \beta_{k}(x)\right)\right\}, \mathbf{z}=\left(z_{1}, \ldots, z_{g}\right) \in \mathbf{C}^{g}$, для всех $\boldsymbol{x} \in \mathbf{R}_{+}, k=1, \ldots, g$ аналитичны в области

$$
\underset{k=1}{\stackrel{g}{\times}}\left\{z_{k}: 0<\operatorname{Re} z_{k}<a_{k}\right\} \subset \mathbf{C}^{g},
$$

при $\mathbf{z} \in(\mathbf{0}, \mathbf{a})=\underset{k=1}{\stackrel{g}{\times}}\left(0, a_{k}\right) \subset \mathbf{R}^{g}$ справедливы неравенства

$$
0<\mathbf{E} \exp \left\{-\left(\mathbf{z}, \boldsymbol{\beta}_{\boldsymbol{k}}(x)\right)\right\}<1, \quad k=1, \ldots, g, x \in \mathbf{R}_{+},
$$

u отображение

$$
(\mathbf{0}, \mathbf{a}) \longrightarrow \mathbf{R}^{g}: \mathbf{z} \longrightarrow\left(\mathbf{E} \exp \left\{-\left(\mathbf{z}, \boldsymbol{\beta}_{1}(1)\right)\right\}, \ldots, \mathbf{E} \exp \left\{-\left(\mathbf{z}, \boldsymbol{\beta}_{g}(1)\right)\right\}\right)
$$

невырождено (взаимно однозначно) в окрестности хотя бы одной точки $\mathbf{z}^{*} \in$ $(\mathbf{0}, \mathbf{a})$. Тогда отображение $\Pi_{B}$, определенное формулой (6), является изоморфизмом между полугруппой $P^{g}$ всех распределений ка $\mathbf{R}_{+}^{g}$ u образом полугруппь $P^{g}$ при отображении $\Pi_{B}$, непрерывным относительно слабой сходимости распределений.

Д ок а з ат т льств о. Как и в теореме 1 , сначала убедимся в том, что отображение $\Pi_{B}$ взаимно однозначно.

Пусть $\gamma=\left(\gamma_{1}, \ldots, \gamma_{g}\right)$ - случайный вектор с неотрицательными координатами, имеюший распределение $F_{\gamma} \in P^{g}$; его преобразование Лапласа $f_{\gamma}(\mathbf{z})=\mathbf{E} \exp \{-(\mathbf{z}, \gamma)\}$ яляется аналитической функцией в области $\left\{\mathbf{z} \in \mathbf{C}^{g}: \operatorname{Re} z_{k} \geqslant 0, k=1, \ldots, g\right\}$. В силу (6) и свойств семейства $B$ распределение $\Pi_{B} F_{\gamma}$ - это распределение случайного вектора $\nu=\beta_{1}\left(\gamma_{1}\right)+\cdots+\beta_{g}\left(\gamma_{g}\right)$, где случайный вектор $\gamma$ и случайные процессы $\beta_{1}(x), \ldots, \beta_{g}(x)$ со значениями в $\mathbf{R}^{g}$ независимы.

По свойству однородных процессов с независимыми приращениями

$$
\mathbf{E} \exp \left\{-\left(\mathbf{z}, \boldsymbol{\beta}_{k}(x)\right)\right\}=\left(\mathbf{E} \exp \left\{-\left(\mathbf{z}, \beta_{k}(1)\right)\right\}\right)^{x}, \quad x \in \mathbf{R}_{+}, k=1, \ldots, g
$$

и так как по условию $0<\mathbf{E} \exp \left\{-\left(\mathbf{z}, \beta_{k}(x)\right)\right\}<1$ при $\mathbf{z} \in \underset{k=1}{\stackrel{g}{\times}}\left(0, a_{k}\right) \subset \mathbf{R}^{g}, x \in \mathbf{R}_{+}$, $k=1, \ldots, g$, то

$$
\begin{aligned}
& \mathbf{E} \exp \{-(\mathbf{z}, \nu)\}=\mathbf{E} \mathbf{E}\left\{\exp \left\{-\left(\mathbf{z}, \beta_{1}\left(\gamma_{1}\right)+\cdots+\boldsymbol{\beta}_{g}\left(\gamma_{g}\right)\right)\right\} \mid \gamma\right\} \\
& =\mathbf{E} \prod_{k=1}^{g} \mathbf{E}\left\{\exp \left\{-\left(\mathbf{z}, \boldsymbol{\beta}_{k}\left(\gamma_{k}\right)\right)\right\} \mid \gamma\right\}=\mathbf{E} \prod_{k=1}^{g}\left(\mathbf{E} \exp \left\{-\left(\mathbf{z}, \beta_{k}(1)\right)\right\}\right)^{\gamma_{k}} \\
& =\mathbf{E} \exp \left\{-\left(\left(-\ln \mathbf{E} \exp \left\{-\left(\mathbf{z}, \beta_{1}(1)\right)\right\}, \ldots,-\ln \mathbf{E} \exp \left\{-\left(\mathbf{z}, \boldsymbol{\beta}_{g}(1)\right)\right\}\right), \gamma\right)\right\} \\
& =f_{\gamma}\left(-\ln \mathbf{E} \exp \left\{-\left(\mathbf{z}, \beta_{1}(1)\right)\right\}, \ldots,-\ln \mathbf{E} \exp \left\{-\left(\mathbf{z}, \boldsymbol{\beta}_{g}(1)\right)\right\}\right) .
\end{aligned}
$$

Согласно условиям теоремы преобразование Лапласа $\mathbf{E} \exp \{-(\mathbf{z}, \nu)\}$ случайной величины $\nu=\beta_{1}\left(\gamma_{1}\right)+\cdots+\beta_{g}\left(\gamma_{g}\right)$ определено в области вида $\left\{0<\operatorname{Re} z_{k}<a_{k}, k=1, \ldots, g\right\}$ и является в ней аналитической функцией. В силу (7) значения аналитической функции $\mathbf{E} \exp \{-(\mathbf{z}, \nu)\}$ в действительной окрестности точки $\mathbf{z}^{*}$ функционально связаны со значениями аналитической функции $f_{\gamma}(\mathbf{z})$ на множестве аналогичного вида и, следовательно (см. [4, с. 286]), каждая из этих функций однозначно определяет другую во всей области ее аналитичности. Отсюда и из взаимной однозначности соответствия между случайными векторами и их преобразованиями Лапласа следует, что отображение $\Pi_{B}$ взаимно однозначно. Непрерывность отображения $\Pi_{B}$ следует из теоремы непрерывности для характеристических функций многомерных распределений (см., например, $[3$, с. 63]). Теорема доказана. 


\section{СПИСОК ЛИТЕРАТУРЫ}

1. Хинчия А.Я. Математические методы теории массового обслуживания. - Труды Матем. ин-та им. В.А. Стеклова АН СССР, 1955, т. 49, с. 1-122.

2. Redheffer R. M. A note on the Poisson law. - Math. Mag., 1953, v. 26, № 4, p. 185188.

3. Kallenberg $O$. Foundations of Modern Probability. Berlin-Heidelberg: SpringerVerlag, 1997, $523 \mathrm{p}$.

4. Шабат Б. В. Введение в комплексный анализ. М.: Наука, 1969, 576 с.

5. Феллер В. Введение в теорию вероятностей и ее приложения, т. 2. М.: Мир, 1984, $752 \mathrm{c}$.

Поступила в редакцию 26.I.1999

(C) $1999 \mathrm{r}$.

МАЦАК И. К.*

\section{О ЗАКОНЕ ПОВТОРНОГО ЛОГАРИФМА} B БАНАХOBЫX PEШETKAX

Для некоторых банаховых решеток устанавливается закон повторного логарифма в классической форме

$$
\limsup _{n \rightarrow \infty} \frac{X_{1}+X_{2}+\cdots+X_{n}}{(2 n \ln \ln (n))^{1 / 2}}=\mathfrak{G} X .
$$

Ключевые слова и фразы: независимые случайные элементы, банаховы решетки, закон повторного логарифма.

1. Введение. Основные результаты. Рассмотрим $\left(\xi_{i}\right), i \geqslant 1$, - последовательность независимых случайных величин (н.с.в.), $\mathbf{E} \xi_{i}=0, \mathbf{E} \xi_{i}^{2}=b_{i}^{2}, B_{n}=\sum_{i}^{n} b_{i}^{2}$. Говорят, что последовательность $\left(\xi_{i}\right)$ удовлетворяет закону повторного логарифма (ЗПЛ), если почти наверное (п.н.)

$$
\limsup _{n \rightarrow \infty} \frac{\xi_{1}+\xi_{2}+\cdots+\xi_{n}}{\left[2 B_{n} L\left(L\left(B_{n}\right)\right)\right]^{1 / 2}}=1, \quad \liminf _{n \rightarrow \infty} \frac{\xi_{1}+\xi_{2}+\cdots+\xi_{n}}{\left[2 B_{n} L\left(L\left(B_{n}\right)\right)\right]^{1 / 2}}=-1,
$$

где $L(t)=\max (1, \ln (t)), t>0$.

Впервые ЗПЛ для некоторого частного случая был установлен А.Я. Хинчиным [1]. Фундаментальную теорему А. Н. Колмогорова и обзор дальнейших исследований ЗПЛ можно проследить по работам [2]-[4].

В общем случае для последовательности $\left(x_{n}\right)$ элементов банахова пространства не существует верхнего и нижнего предела. По-видимому, это и послужило одной из причин того, что в банаховых пространствах рассматривались различные непрямые обобщения ЗПЛ (1) (так называемые ограниченный и компактный ЗПЛ) [5]. Но понятия $\lim \sup _{n \rightarrow \infty} x_{n}$ и $\liminf _{n \rightarrow \infty} x_{n}$ можно ввести в банаховых решетках (и даже в векторных решетках). Кроме того, в банаховых решетках можно рассматривать среднее квадратическое отклонение случайного элемента (с.э.). Поэтому естественно ставить задачу непосредственного обобщения равенств (1) на с.э. банаховой решетки. Этой задаче и посвящена наша работа.

Далее через $E$ будем обозначать сепарабельную банахову решетку с нормой $\|\cdot\|$ и модулем $|\cdot|$. Следуюшие ниже определения и используемые результаты из теории банаховых решеток можно найти в книгах [6]-[8].

* Государственная академия легкой промышленности Украины, кафедра вычислительной техники и прикладной математики, ул. Немировича-Данченко, 2, 252011 Киев, Украина. 